\title{
COVID-19 and rare diseases: reflections and recommendations by the International Rare Diseases Research Consortium
}

\author{
Chiuhui Mary Wang ${ }^{1}$, Daria Julkowska ${ }^{2}$, Chun-Hung Chan ${ }^{3}$, David A. Pearce ${ }^{3,4,5}$, Lucia Monaco \\ ${ }^{1}$ Fondazione Telethon, Milan 20129, Italy. \\ ${ }^{2}$ INSERM, Hôpital Charles Foix, Paris 75013, France. \\ ${ }^{3}$ Sanford Research, Sioux Falls, SD 57104, USA. \\ ${ }^{4}$ Sanford Health, Sioux Falls, SD 57104, USA. \\ ${ }^{5}$ Sanford School of Medicine, Sioux Falls, SD 57104, USA. \\ Correspondence to: Dr. David A. Pearce, Sanford Health, 2301 E 60th Street N, Sioux Falls, SD 57104, USA. \\ E-mail: david.pearce@sanfordhealth.org
}

How to cite this article: Wang CM, Julkowska D, Chan CH, Pearce DA, Monaco L. COVID-19 and rare diseases: reflections and recommendations by the International Rare Diseases Research Consortium. Rare Dis Orphan Drugs J 2021;1:4.

https://dx.doi.org/10.20517/rdodj.2021.03

Received: 29 Sep 2021 First Decision: 19 Nov 2021 Revised: 8 Dec 2021 Accepted: 16 Dec 2021 Published: 27 Dec 2021

Academic Editor: Daniel Scherman Copy Editor: Xi-Jun Chen Production Editor: Xi-Jun Chen

\begin{abstract}
Aim: The ambitious goals set by the International Rare Diseases Research Consortium (IRDiRC) by 2027 to fulfill the vision of providing diagnosis and treatments to rare diseases (RDs) patients within one year of coming to medical attention have been challenged by the COVID-19 pandemic. This article aims to identify the needs and challenges of the RD community during the COVID-19 pandemic and to understand whether the pandemic would hinder achievement of the IRDiRC goals.
\end{abstract}

Methods: A survey was developed in 2020 to answer key issues related to the potential impact of the pandemic on RD research and distributed to all 96 IRDiRC Constituent Committee members and Scientific Committee experts.

Results: The overall participation rate was $46 \%$, with the highest response rates from the Patient Advocates, Funders, and Therapies Committees. Most respondents reported impacts on various aspects of RD research including decreased access to healthcare, clinical trials, and diagnostics for patients, as well as disrupted operations for patient and funding organizations and restrictions in access to workplaces for researchers. Despite these challenges, there was overall optimism that the IRDiRC goals could still be met by 2027, although there 
would be an inevitable slowdown in RD research activities.

Conclusions: Maintaining funding for RD research and implementing new workflows to ensure that patients have continued access to diagnostics, therapies, and clinical trials will be key to ensuring that IRDiRC meets it goals by 2027.

Keywords: IRDiRC, rare diseases, COVID-19, survey, recommendations

\section{INTRODUCTION}

Although the COVID-19 pandemic's effects on biomedical research and patient communities have been widely examined ${ }^{[1-7]}$, here we report the outcome of a survey launched by the International Rare Diseases Research Consortium (IRDiRC) among its members and scientific experts. The survey provides a unique combined perspective of IRDiRC's key stakeholders, namely funders, patients, and companies, united in the research effort to tackle rare diseases (RDs) globally.

People living with a RD, estimated as 263-446 million worldwide, experience a condition of fragility, due to lack of appropriate treatments for most of the estimated 6000-10,000 $\mathrm{RDs}^{[8,9]}$. The United Nations recommended strengthening efforts to address RDs, as they still require global development efforts to generate sustainable health solutions ${ }^{[10]}$.

Launched in 2011, today IRDiRC gathers 59 member organizations supported by the expertise of three scientific committees ${ }^{[1,1,2]}$. IRDiRC already demonstrated that by global, collaborative efforts ambitious goals could be achieved and in 2017 set up an aspiring vision of enabling all people living with a RD to receive an accurate diagnosis, care, and available therapy within one year of coming to medical attention ${ }^{[12-14]}$. Three goals were set for 2027, addressing the urgent need for diagnoses and therapies, as well as the need to measure the impact of these solutions on patients and families ${ }^{[15]}$. IRDiRC members and experts are actively engaged in task forces tackling specific topics aimed at developing solutions to achieve these goals ${ }^{[16]}$.

When COVID-19 began to significantly impact societies across the world, IRDiRC members and scientific experts were surveyed about how, in their view, the crisis would hinder the achievement of IRDiRC goals, and whether IRDiRC should address COVID-19 through dedicated actions. The survey (conducted between 18 June and 4 August 2020) collected 44 responses out of 96 members and experts [Table 1, Figure 1].

The goal of the survey was to gauge the impact of COVID-19 on the RD research community and to understand how IRDiRC can react to such changes. Key questions were the following:

1. Is the crisis going to hinder or delay achievement of IRDiRC goals?

2. Are there new and unforeseen issues that have arisen that IRDiRC could address through dedicated actions (task forces, working groups)?

3. What requests or needs by the RD research community can IRDiRC convey to international authorities or bodies?

\section{METHODS}

\section{Participants of the survey}

The target participants of the survey were all 96 IRDiRC Constituent Committee members and Scientific Committee experts in 2020 [Figure 1]. The Constituent Committee members are funders, companies, or umbrella patient advocate organizations who have joined IRDiRC. The scientific experts are persons 
Table 1. IRDiRC COVID-19 survey respondents

\begin{tabular}{|c|c|}
\hline $\begin{array}{l}\text { COVID-19 Survey } \\
\text { conducted: }\end{array}$ & 18 June- 4 August 2020 \\
\hline Responses: & 44 out of 96 members and experts ( $46 \%$ response rate) \\
\hline $\begin{array}{l}\text { Responses from countries } \\
\text { (or regions): }\end{array}$ & $\begin{array}{l}\text { Australia, Belgium, Canada, China, France, Georgia, Germany, India, Israel, Italy, Japan, Netherlands, Saudi Arabia, } \\
\text { Spain, UK, USA, Asia Pacific, Europe, and Latin America representatives }\end{array}$ \\
\hline
\end{tabular}

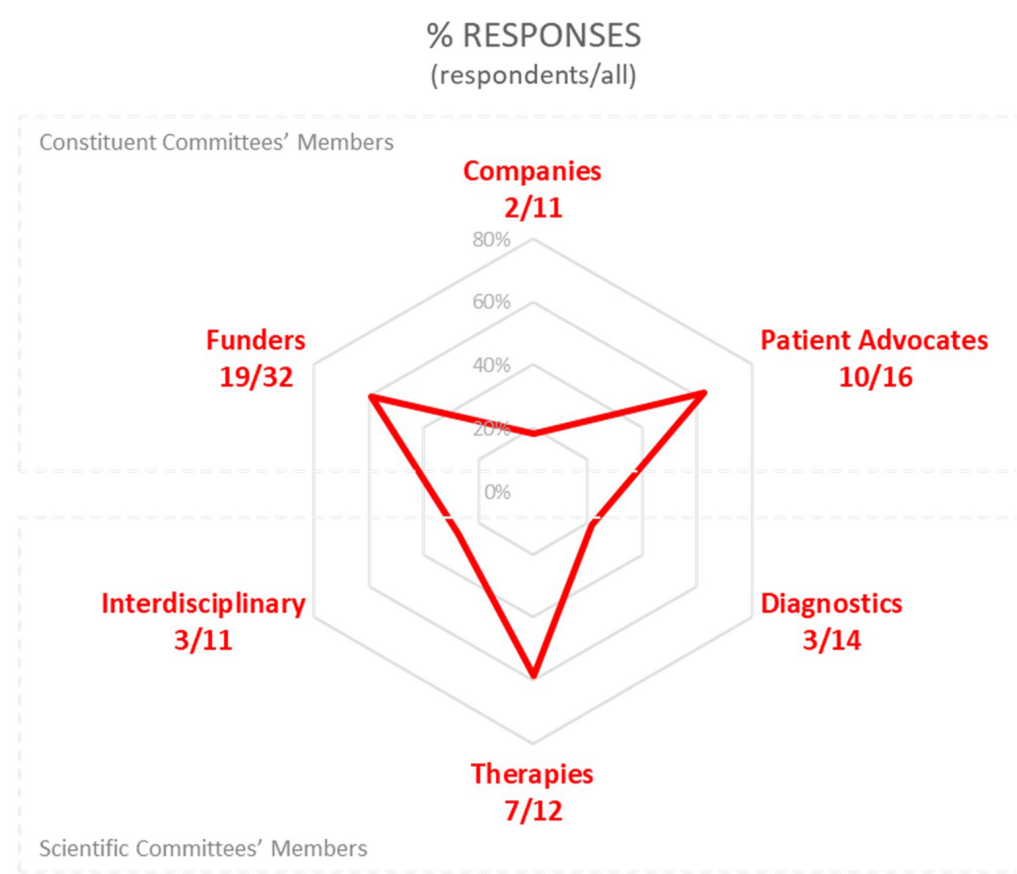

Figure 1. Distribution of respondents to the survey and their affiliations to IRDiRC Constituent and Scientific Committees. The response rate from the Patient Advocates Constituent Committee (PACC, 63\%), Funders Constituent Committee (FCC, 59\%), and Therapies Scientific Committee (TSC, 58\%) were the highest, with an overall participation rate of 46\%. IRDiRC: International Rare Diseases Research Consortium.

nominated and elected by the IRDiRC Consortium Assembly who have specific expertise in areas of rare diseases diagnosis, therapy, or interdisciplinary sciences. The IRDiRC members and experts are located across the globe $e^{[12]}$.

\section{Setup of the survey}

The survey contained three parts: (1) name and country location of the survey participant; (2) questions on the impact of COVID-19 on IRDiRC goals; and (3) specific questions on the impact the pandemic on the activities carried out by Constituent Committees' member organization. Parts (1) and (2) contained questions for all participants, whereas Part (3) questions were addressed to members of the three specific IRDiRC Constituent Committees (Funders, Companies, or Patient Advocates, respectively). All questions were compulsory except for free text spaces for optional explanations or comments.

\section{Data collection}

The survey was set up as an online questionnaire using Microsoft Forms. The survey was sent to the IRDiRC members and experts on 18 June 2020. The participants were allowed to complete the form in their own time until 4 August 2020, and the form was subsequently taken offline. 


\section{Analysis}

The survey responses were exported as a table in Microsoft Excel and descriptive statistics were performed. All graphs were created using Microsoft Excel. The final qualitative analysis was based on the 44 survey responses, as well as on the discussion that followed the presentation of the survey results during the 20th IRDiRC Consortium Assembly on 2 December $2020^{[17]}$.

\section{RESULTS}

Impact on clinical trials and access to treatments

Patient Advocacy Organizations (PAO) are important resources for RD patients and their families and, as such, often have a close relationship with their members. Therefore, PAOs were surveyed to obtain data on the potential effects of the pandemic on RD patients. The major observations collected confirmed that the impact on RDs is real. Patients were heavily affected and experienced impaired access to regular care, clinical trials, and diagnostic paths. The perceived societal impact was high, affecting patients, caregivers, and families.

Rare disease patients experienced issues in accessing diagnostic paths and therapies due to the pandemic [Figure 2A]. Both Patient Advocacy Constituent Committee (PACC) and Company Constituent Committee (CCC) perceived impacts on rare diseases clinical trials.

In particular, companies reported impact on their usual R\&D processes, highlighting issues in the initiation of new trials and review/approval process. Management of ongoing clinical trials focused on rare diseases was hampered in regard to availability of on-site monitoring visits and patient enrollment/retention. Similarly, rare disease PAOs were aware of delays in the starting of trials or postponement of trials. In response, several alternative strategies were adapted by companies for ongoing clinical trials such as remote monitoring and remote enrollment to enable these trials to continue.

\section{Impact on PAOs}

PAOs are not only important resources for patients and their caregivers but also important conduits for physicians and researchers who work towards developing new therapies and treatments for $\mathrm{RD}$ patients. As such, the ability of the PAOs to continue their work is essential for advancing RD patient care and PACC members reported disruptions in their organizations as a result of COVID-19 [Figure 2B]. These included challenges in fundraising and cancellation of events, increased demand for information/support, and a reduced capacity for participation in, or support for, research activities.

\section{Impact on funding for rare disease research}

Research and development on RDs were reported as hampered, delayed, or reduced by IRDiRC Funder organizations. Importantly, both research efforts and funding were often diverted towards COVID-19. Several funders expressed uncertainty about future financial resources. Many funders reported an impact on opening of calls for applications, anticipated duration of projects, and receiving submissions for funding [Figure 3A]. For active grants, many funders received requests to extend project deadlines, suspend (temporarily) grants, and requests for additional funding [Figure $3 \mathrm{~B}$ ]. In response to these challenges, many organizations implemented measures that included extending deadlines for submissions, joining other organizations/agencies to offer new funding opportunities, and allowing delayed start of funding [Figure 3C].

\section{Organizational responses towards the pandemic}

In response to the pandemic, several IRDiRC member organizations launched their own surveys to understand and map the needs of their communities and stakeholders [Figure $4 \mathrm{~A}$ and $\mathrm{B}$ ]. 
A

\section{Have you, as a Patient Advisory Organization, become aware of specific problems experienced by RD patients caused by the pandemic that are hindering accomplishment of IRDiRC goals?}

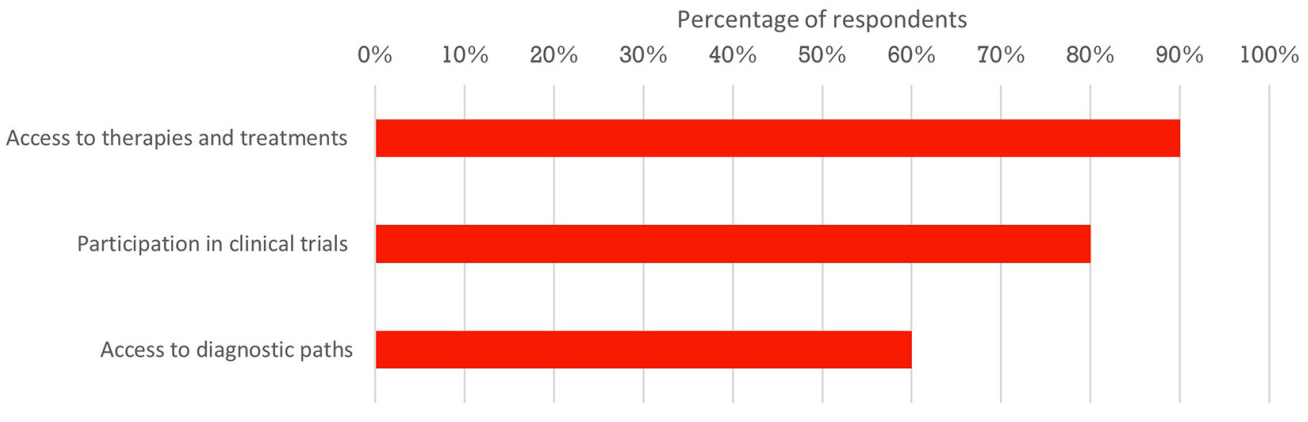

B

Did you perceive any impact on your organization that is due to the COVID-19 pandemic?

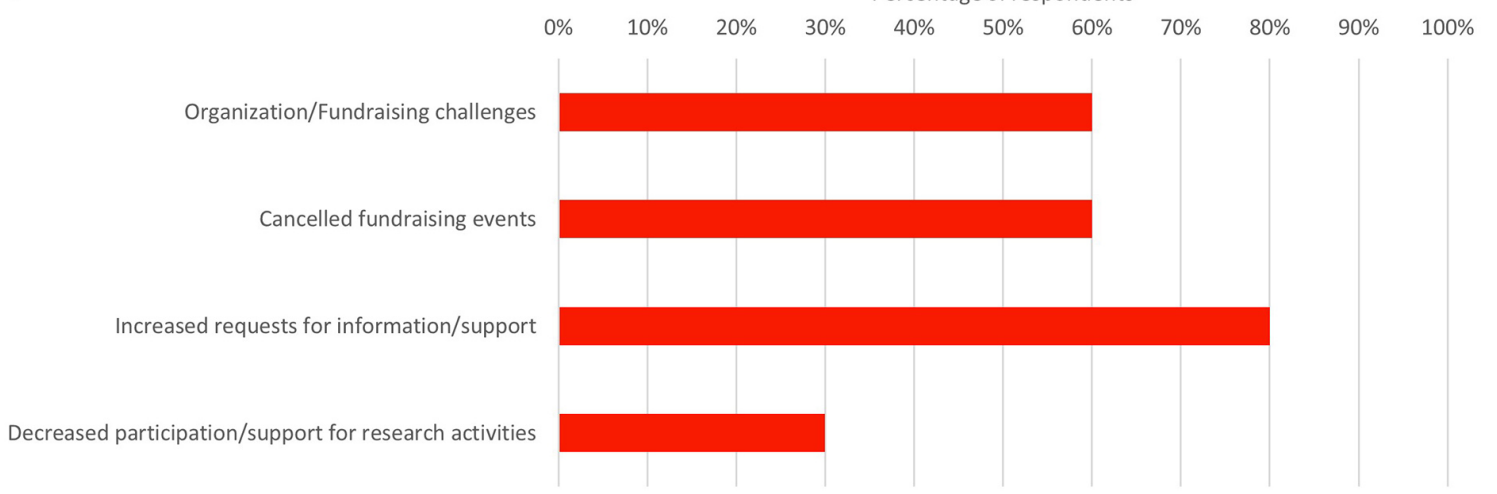

Figure 2. Patient Advocacy Organizations reporting impacts of the COVID-19 pandemic on their organization. The PAOs reported that their members were negatively impacted by the pandemic as a result of decreased access to treatments, clinical trials, and access to diagnostic paths (A). In addition, PAOs reported challenges in operations resulting from increased demand for information/support, cancellation of fundraising events, and decreased participation and support for research activities (B). PAOs: Patient Advocacy Organizations.

Many IRDiRC member organizations reported that funding opportunities focused towards COVID-19 were made available during the pandemic [Figure 5].

Are we on track to achieve IRDiRC Goals by 2027?

The the goals of IRDiRC for 2017-2027 are as follows:

1. All patients coming to medical attention with a suspected rare disease will be diagnosed within one year if their disorder is known in the medical literature; all currently undiagnosable individuals will enter a globally coordinated diagnostic and research pipeline.

2. One thousand new therapies for rare diseases will be approved, the majority of which will focus on diseases without approved options. 


\section{A Did you experience any impact on any steps of your usual granting process for rare diseases calls due to the COVID-19 pandemic?}

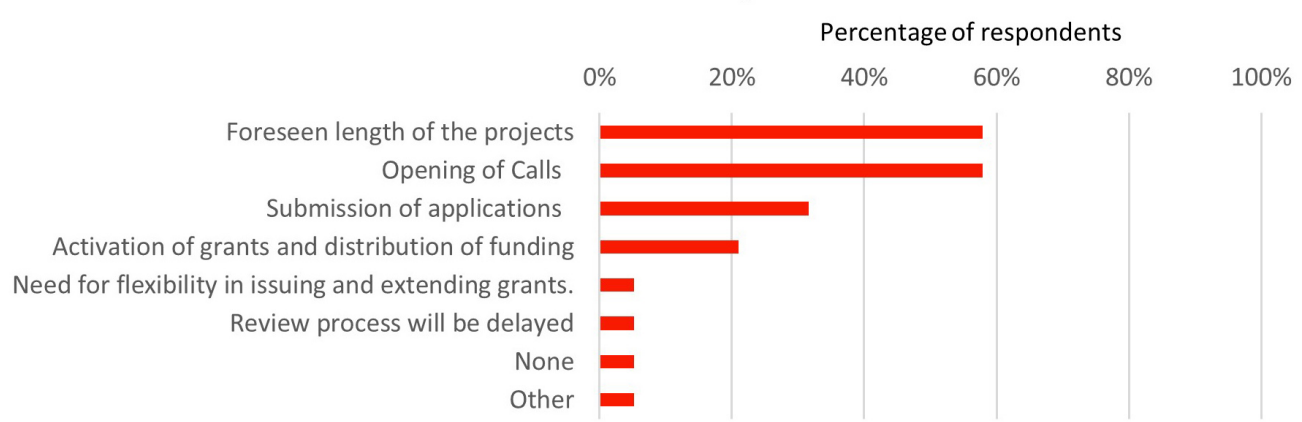

\section{B Did you experience any of the following in managing existing rare disease grants due to the COVID-19 pandemic?}

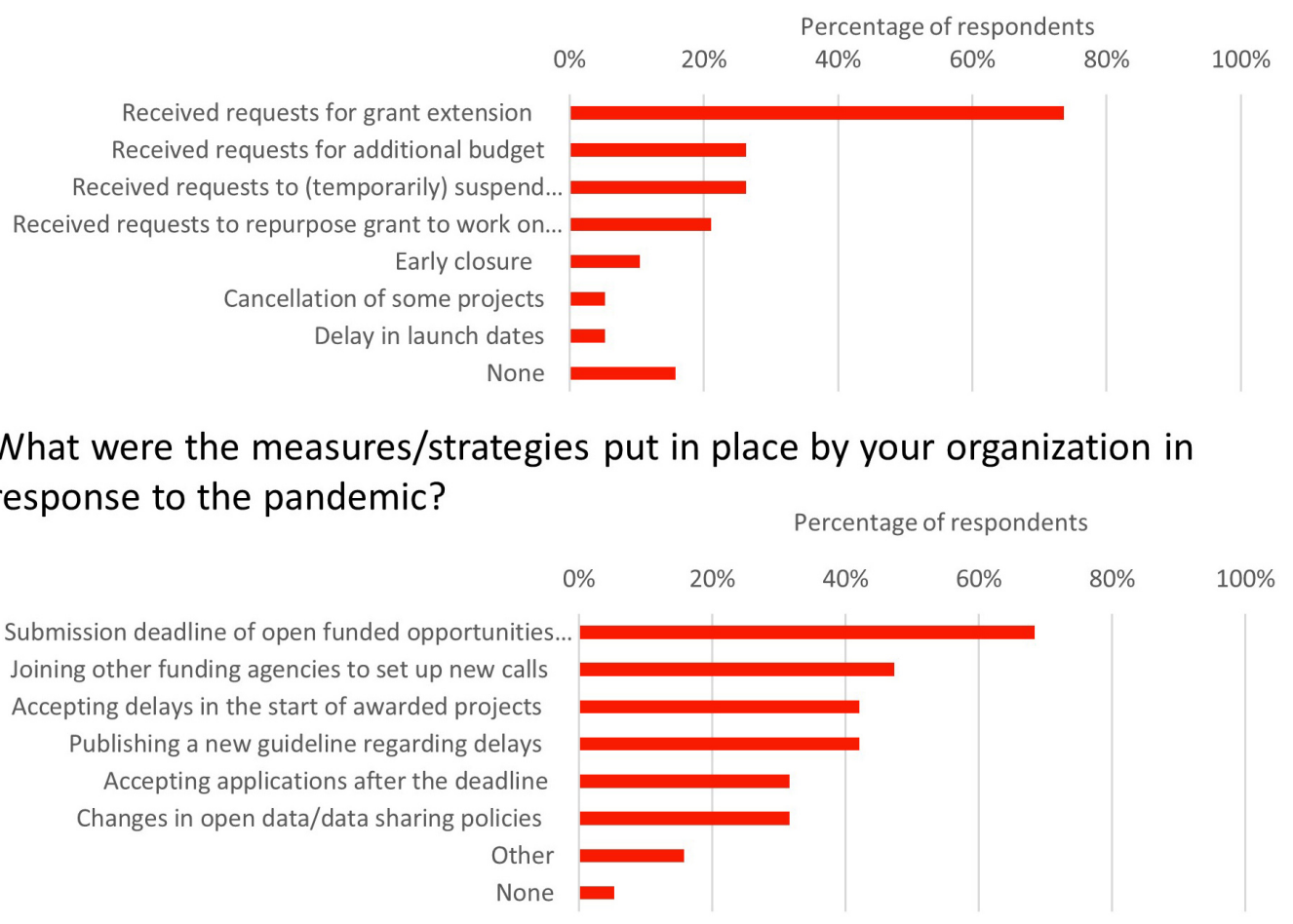

Figure 3. Impact on funding to support rare disease research. Respondents reported impacts on their normal processes for awarding grant funding (A) and received requests for extensions to existing grants, additional funding, and suspension of current grants (B). In response, many organizations offered more flexibility in grant submission deadlines and acceptance, as well as exploring opportunities to partner with other agencies to offer new calls for application (C).

3. Methodologies will be developed to assess the impact of diagnoses and therapies on rare disease patients.

Overall, most respondents felt that the IRDiRC goals could still be met by 2027 despite the on-going pandemic [Figure 6], with one respondent stating "there is considerable momentum in the rare disease community, especially for therapeutics and gene therapies. COVID-19 will slow us down, but things should get back on track thereafter". 
A Have you or your organization launched a survey or collected information related to the COVID-19 pandemic from across your stakeholders?

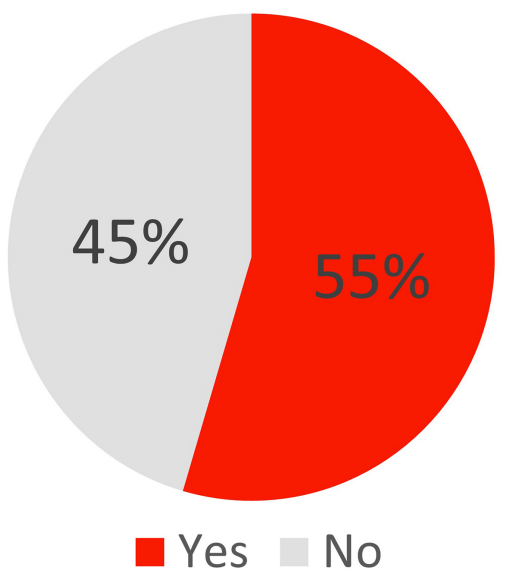

B Would you be willing to share key findings relevant to an envisaged impact on achievement of IRDiRC goals by 2027 ?

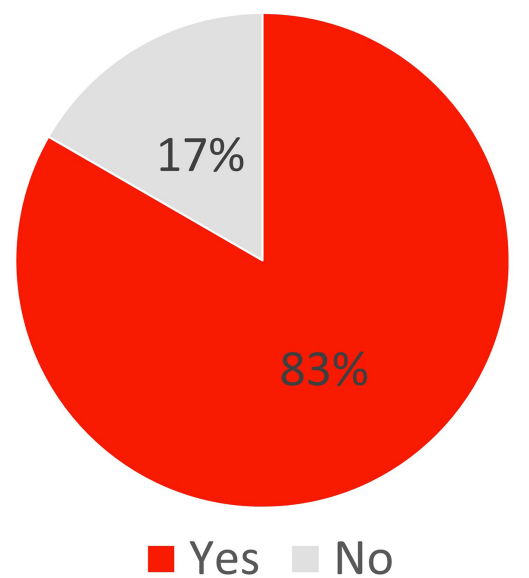

Figure 4. Member organizations who responded to the COVID-19 pandemic by surveying key stakeholders. Over half of the organizations surveyed reported conducting their own survey of key stakeholders (A). Of those, the majority were willing to share their findings (B).

\section{Did your organization decide to open a COVID-19 related call?}

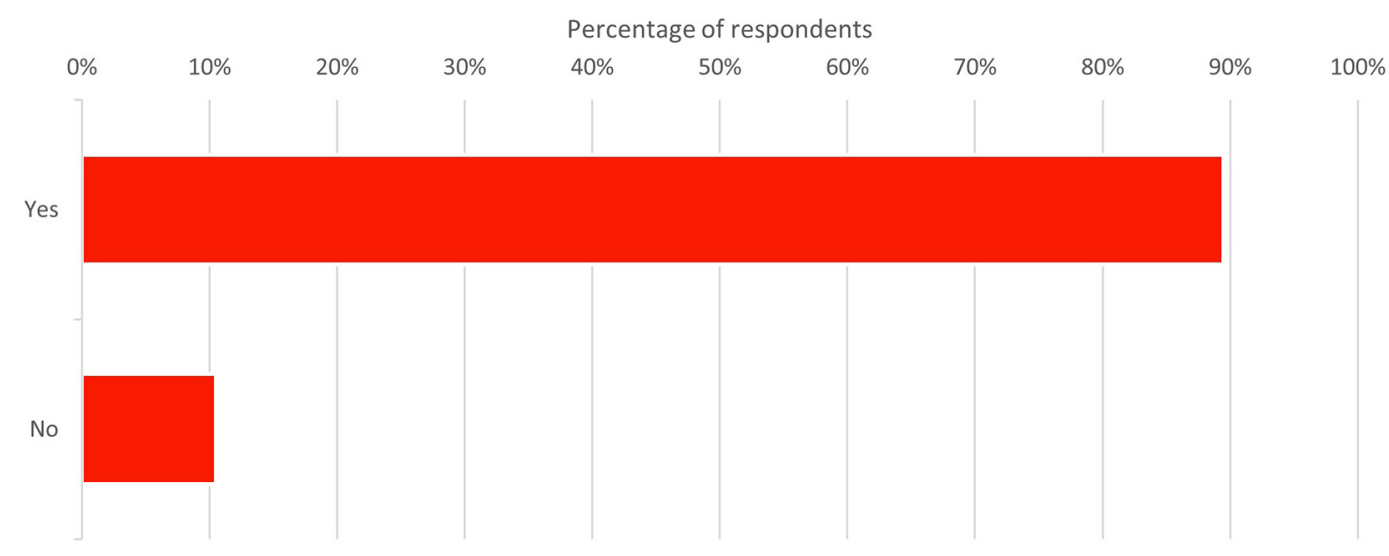

Figure 5. IRDiRC Funder members who allocated resources towards COVID-19 related activities.

All respondents forecasted a slowdown in the progress towards IRDiRC goals, although it was difficult to foresee whether it would be possible to recover by 2027, or whether a 1-2-year delay should be envisaged. A general concern regarded the re-prioritization of investment and resources away from RD research towards combating the COVID-19 pandemic, and the foreseeable reduction in general investments in research, due to the economic crisis caused by the pandemic. This concern is encapsulated in this quote from one respondent: "SARS-CoV-2 outbreak has seriously impacted ongoing research and clinical trials in rare diseases, as well as re-routed the priority and focus of researchers, companies, funders, and regulators toward fighting CoViD-19 disease".

On the optimistic side, several respondents envisaged positive spillover effects from the pandemic, namely faster clinical trials and drug development processes, expansion of telemedicine and digital tools, enhanced sharing of knowledge (pre-publications), and scientific collaborations. 


\section{Do you think IRDiRC goals can be achieved on time by 2027 despite the COVID-19 global outbreak?}

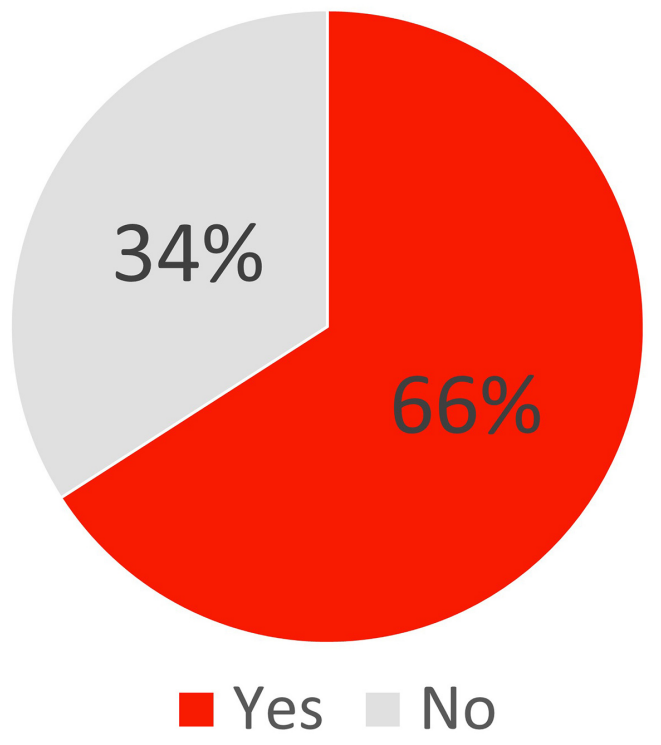

Figure 6. IRDiRC members were asked whether the COVID-19 pandemic might significantly impact reaching the IRDiRC goals by 2027.

Respondents suggested extracting and using lessons learned on COVID-19, from both RDs and potentially other disease areas, to prepare for the next crisis (pandemic or other) in important areas such as ethical, legal, and social implications, clinical trials and treatment access, research and development, telemedicine, and funding.

\section{Focus on rare disease research}

IRDiRC members were next asked whether IRDiRC should direct specific efforts towards COVID-19. When asked whether a COVID-19 taskforce or working group should be established within IRDiRC, respondents were split [Figure 7A]. One respondent stated: "perhaps it should not be a COVID-19 task force, but rather a task force that looks at modifications due to COVID-19 and guidance around those modifications - telehealth, remote trial participation, supply chain, revisions to trials, etc.".

When asked whether IRDiRC should engage in direct action in response to COVID-19, two-thirds of respondents were supportive of direct actions [Figure 7B]. Suggestions expressed in the written comments were discussed in the Consortium Assembly meeting on 2 December 2020. The Assembly agreed on the need to stay focused on the IRDiRC goals while taking advantage of lessons learned from the COVID-19 experience and relying on the several COVID-19 surveys performed by individual member organizations.

\section{DISCUSSION}

It is evident from the responses received to our survey that the global COVID-19 pandemic has had a profound impact on all facets of rare diseases, from patient access to diagnostics and therapies through to research efforts directed towards improving patient care. Besides the impact on patients, IRDiRC members expressed concern over the pandemic's effect on research resulting from re-allocation of resources and funding, disruptions to work due to COVID-19 preventative measures and illness, delays in grant allocations, and inability to conduct clinical trials. These outcomes are in accord with the general scenario that emerged with the pandemic. In Europe alone, more than $€ 1$ billion has been allocated from the Horizon 

A Do you see a need for a dedicated COVID-19 IRDiRC Task Force or Working Group?

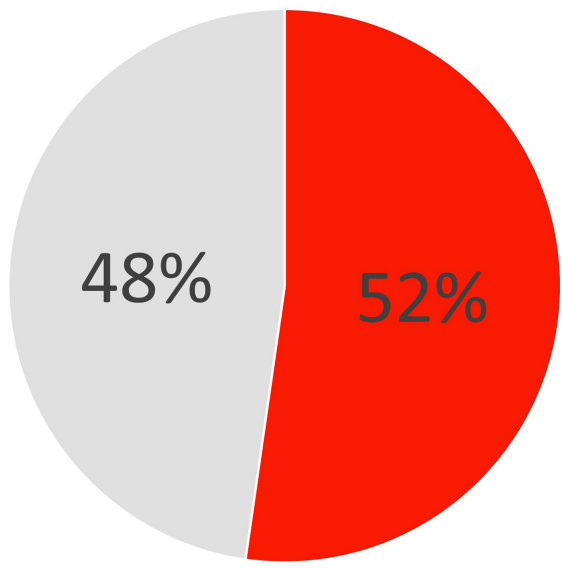

$\square$ Yes $\square$ No
B

Would you like to see IRDiRC engaging in an action on COVID-19?

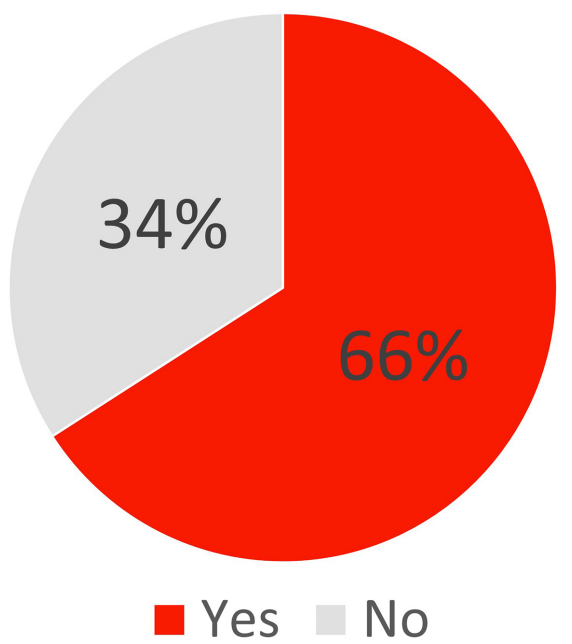

Figure 7. IRDiRC members were divided over the need for a specific COVID-19 task force or working group (A), but the majority would consider devoting specific efforts towards COVID-19 (B).

2020 to tackle the COVID-19 pandemic ${ }^{[18]}$, while, in the United States, over $\$ 4.9$ billion has been committed $^{[19]}$. The detrimental impact on rare disease research is not unexpected given that the COVID-19 pandemic has had a significant impact on global health research as a whole ${ }^{[1-4]}$. However, rare diseases are often neglected due to low numbers of patients and priority for pharmaceutical companies, so it is imperative that the research community and funding agencies do not overlook rare diseases as the world begins to return to pre-pandemic activities.

As anticipated, COVID-19 had a significant impact on all aspects of biomedical research, as evidenced by multiple surveys conducted during the pandemic ${ }^{[1,5-7]}$. Many respondents in those surveys reported disruptions in the workplace and uncertainty over funding which correlates well with IRDiRC survey respondents who reported increased requests for extension of grant funding and additional funding, as well as temporary suspension or early closure of existing funding, mainly as a result of workplace disruptions. Additionally, COVID-mitigation efforts such as lockdowns likely not only resulted in workplace disruptions but also created barriers for patients to obtain care, which was reflected in responses to the IRDiRC survey indicating reduced access to therapies and treatments, clinical trials, and diagnostics experienced by patients.

Based on these observations, we recommend that private and public funding bodies maintain investment in RDs and take advantage of advancements spurred by the COVID-19 pandemic to stimulate even better research for the benefit of $\mathrm{RD}$ patients. We urge companies to maintain priority on $\mathrm{RD}$ clinical trials and development paths and healthcare providers to facilitate patient participation in clinical studies and remove barriers to the available diagnosis and care for RD patients.

Despite the challenges posed by the COVID-19 pandemic, in general, IRDiRC members felt confident that the three stated goals were still achievable by 2027 if funding is maintained for RD research and healthcare providers implement new workflows to ensure that patients have continued access to diagnostics, therapies, and clinical trials. The first goal of having all patients with a suspected rare disease receiving a diagnosis within one year is achievable if research is maintained and healthcare centers remain accessible to patients. 
The impact of COVID-19 on this goal, with reduced access to research funding, restrictions on ability to access workplaces, and reduced mobility of patients to seek medical care, was significant but can be overcome as the world emerges from the shadow of the pandemic and begins to return to pre-pandemic levels of activity. The second goal of 1000 new therapies between 2017 and 2027 is still within reach if research funding is maintained and research efforts are re-focused on rare diseases if they were previously diverted toward the fight against COVID-19. During the pandemic, many clinical trials faced challenges in enrollment, patient access, hospital capacity, staffing, supply chain issues, and sponsor monitoring and oversight. In addition, resources were often re-allocated to clinical trials for COVID-19, thereby reducing the ability to conduct critical clinical trials for rare diseases. As we emerge from the COVID-19 pandemic, it will be important to ensure that resources are devoted to new and existing clinical trials to obtain the data required for new approvals. Finally, the third goal of developing methodologies to assess the impact of diagnoses and therapies on rare diseases can be achieved, as this goal was least impacted by the pandemic. Stakeholders were able to pivot to new ways to work and collaborate, with increasing use of teleconferencing to link collaborators remotely and continue the important work toward this goal. The outcome of the IRDiRC survey illustrated in this article stimulated discussion within the IRDiRC Consortium Assembly, leading to the reinforced intention to advance on the goals set for 2027, according to the annually established roadmap, while monitoring relevant issues, including the pandemic, and elaborating on needs and opportunities within the activities of its stakeholders.

Undoubtedly, COVID-19 has added fragility to the health condition of people living with a $\mathrm{RD}$ and put the efforts of researchers, healthcare providers, and therapy developers at stake. Nevertheless, it also made people understand the daily struggle of the RD condition: isolation, fear, lack of knowledge, lack of available or accessible diagnosis and treatments, and economic fragility. Ever more, IRDiRC is here to support the $\mathrm{RD}$ community with guidance, tools, and actions to advance research and healthcare practice to the benefit of all those living with a rare disease.

\section{DECLARATIONS}

\section{Acknowledgements}

The authors wish to thank Galliano Zanello and Carla D'Angelo for their work in developing and administering the survey whose results are presented here. The authors wish to thank the IRDiRC members for responding to the survey and discussion during the Consortium Assembly meeting.

\section{Author's contributions}

Made substantial contributions to conception and design of the study and performed data analysis and interpretation: Wang CM, Julkowska D, Pearce DA, Monaco L

Performed data acquisition, as well as provided administrative, technical, and material support: $\mathrm{Chan} \mathrm{CH}$

\section{Availability of data and materials}

Not applicable.

\section{Financial support and sponsorship}

This work was supported by the Scientific Secretariat of IRDiRC, funded by the European Union through the European Joint Programme on Rare Disease under the European Union's Horizon 2020 research and innovation programme Grant Agreement ${ }^{\circ} 825575$

\section{Conflicts of interest}

All authors declared that there are no conflicts of interest 


\section{Ethical approval and consent to participate}

Not applicable.

\section{Consent for publication}

Not applicable.

\section{Copyright}

(c) The Author(s) 2021.

\section{REFERENCES}

1. Kabanova A, Gavriilaki E, Pelzer BW, Brunetti L, Maiques-Diaz A. Effect of the COVID-19 pandemic on laboratory and clinical research: a testimony and a call to action from researchers. Hemasphere 2020;4:e499. DOI PubMed PMC

2. Singh JA, Bandewar SV, Bukusi EA. The impact of the COVID-19 pandemic response on other health research. Bull World Health Organ 2020;98:625-31. DOI PubMed PMC

3. Sohrabi C, Mathew G, Franchi T, et al. Impact of the coronavirus (COVID-19) pandemic on scientific research and implications for clinical academic training - a review. Int J Surg 2021;86:57-63. DOI PubMed PMC

4. Weiner DL, Balasubramaniam V, Shah SI, Javier JR; Pediatric Policy Council. COVID-19 impact on research, lessons learned from COVID-19 research, implications for pediatric research. Pediatr Res 2020;88:148-50. DOI PubMed

5. National Institutes of Health. NIH workforce COVID-19 impact survey. Available from: https://diversity.nih.gov/sites/coswd/files/images/docs/25690_Trans-NIH_COVID-19_Executive_Summary_508_v2.pdf [Last accessed on 22 Dec 2021].

6. Myers KR, Tham WY, Yin Y, et al. Unequal effects of the COVID-19 pandemic on scientists. Nat Hum Behav 2020;4:880-3. DOI PubMed

7. National Institutes of Health. 2020 NIH extramural surveys: the impact of COVID-19 on the Research Community. Available from: https://diversity.nih.gov/sites/coswd/files/images/images/NIH_COSWD_COVID19_Impact_Infographic_vF_Updated_3-19.pdf [Last accessed on 22 Dec 2021].

8. Nguengang Wakap S, Lambert DM, Olry A, et al. Estimating cumulative point prevalence of rare diseases: analysis of the Orphanet database. Eur J Hum Genet 2020;28:165-73. DOI PubMed PMC

9. Haendel M, Vasilevsky N, Unni D, et al. How many rare diseases are there? Nat Rev Drug Discov 2020;19:77-8. DOI PubMed PMC

10. United Nations. Universal health coverage: moving together to build a healthier world. Available from: https:/www.un.org/pga/73/wpcontent/uploads/sites/53/2019/07/FINAL-draft-UHC-Political-Declaration.pdf [Last accessed on 22 Dec 2021].

11. IRDiRC. Member Organizations Available from: https://irdirc.org/about-us/people-organisation/members-organizations/ [Last accessed on 22 Dec 2021].

12. Dawkins HJS, Draghia-Akli R, Lasko P, et al; International Rare Diseases Research Consortium (IRDiRC). Progress in Rare Diseases Research 2010-2016: an IRDiRC perspective. Clin Transl Sci 2018;11:11-20. DOI PubMed PMC

13. Cutillo CM, Austin CP, Groft SC. A global approach to rare diseases research and orphan products development: the International Rare Diseases Research Consortium (IRDiRC). In: Posada de la Paz M, Taruscio D, Groft SC, editors. Rare diseases epidemiology: update and overview. Cham: Springer International Publishing; 2017. p. 349-69. DOI PubMed

14. Julkowska D, Austin CP, Cutillo CM, et al. The importance of international collaboration for rare diseases research: a European perspective. Gene Ther 2017;24:562-71. DOI PubMed PMC

15. Austin CP, Cutillo CM, Lau LPL, et al; International Rare Diseases Research Consortium (IRDiRC). Future of rare diseases research 2017-2027: an IRDiRC perspective. Clin Transl Sci 2018;11:21-7. DOI PubMed PMC

16. IRDiRC. Task Forces \& Working Groups. Available from: https://irdirc.org/activities/task-forces/ [Last accessed on 22 Dec 2021].

17. IRDiRC. Minutes of the 20th Consortium Assembly Meeting. Available from: https://irdirc.org/wpcontent/uploads/2020/11/Report_20th-CA-meeting_October-2020.pdf [Last accessed on 22 Dec 2021].

18. European Union. Coronavirus: Commission steps up research funding with $€ 120$ million for 11 new projects to tackle the virus and its variants. Available from: https:/ec.europa.eu/commission/presscorner/detail/en/IP_21_3803 [Last accessed on 22 Dec 2021$].$

19. National Institutes of Health. COVID-19 Funded Research Projects. Available from: https://covid19.nih.gov/funding\#: :text=NIH\%20 has $\% 20$ received $\% 20$ almost $\% 20 \% 244.9$, coming $\% 20$ from $\% 20$ the $\% 20$ U.S. $\% 20$ Congress $\% 20$ [Last accessed on 22 Dec 2021 ]. 